\title{
Perfil e hábitos dos consumidores de cachaça no Estado da Paraíba
}

\section{Profile and habits of consumers of cachaça in the State of Paraíba}

\author{
Renan Elan da Silva Oliveira ${ }^{1 *}$; Manoel José da Silva ${ }^{1}$; Edilma Pinto Coutinho²; \\ Ricardo Targino Moreira ${ }^{3}$; George Rodrigo Beltrão da Cruz ${ }^{4}$
}

\section{Resumo}

O objetivo neste trabalho foi estudar o perfil e os hábitos do consumidor de cachaça no estado da Paraíba. A pesquisa foi descritiva e quantitativa, realizada por meio da aplicação de 595 questionários estruturados nas quatro mesorregiões paraibanas: Litoral, Agreste, Borborema e Sertão. A análise estatística utilizou o teste de Hertley e o teste $t$ de Student a 5\% de probabilidade. Quanto ao gênero dos consumidores de cachaça entrevistados, $84,2 \%$ são masculinos, e $15,8 \%$, femininos. Observou-se que a frequência de consumo diária e semanal é maior entre os consumidores de baixa escolaridade, e os com ensino superior apresentaram o menor consumo diário. Os locais preferidos para a aquisição da cachaça foram os bares $(51,4 \%)$ e os supermercados (26,2\%). Na Mesorregião Agreste, 4,7\% dos consumidores afirmaram que adquirem a cachaça diretamente do produtor. A cachaça de produção industrial foi apontada como a preferida por $50,4 \%$ dos consumidores paraibanos, entretanto, na Mesorregião do Agreste, reduto que produz a cachaça de forma artesanal, os consumidores relataram preferência por cachaça produzida artesanalmenete. Em todo o estado, um percentual elevado de consumidores relatou que não sabe a diferença entre cachaça de produção artesanal e industrial.

Palavras-chave: Bebida alcoólica, consumo de cachaça, cachaça artesanal, cachaça industrial, hábitos de consumo, preferências do consumidor

\begin{abstract}
The objective in this work was to study the profile and the habits of consumers of cachaça in the state of Paraíba. The study was descriptive and quantitative and has been carried out through the application of 595 structured questionnaires in the four paraiban meso-regions: the Coastal region, Agreste, Borborema and the Sertão. For statistical analysis the Hertley test and Student's t-test at 5\% probability were used. As far as the gender of the interviewed consumers of cachaça was concerned, $84,2 \%$ were male and $15,8 \%$ were female. It was observed that the daily and weekly consumption was higher among consumers of low scolarity. The consumers with the highest scolarity presented the lowest daily consumption. The preferred locale for acquisition of cachaça were bars $(51,4 \%)$ and supermarkets $(26,2 \%)$. In the meso-region, Agreste, $4,7 \%$ of the consumers confirmed acquiring cachaça directly from the producer. Industrial cachaça was indicated as the preferred one by $50,4 \%$ of the paraiban consumers, whereas, in the meso-region Agreste, where there is a concentration of artisan cachaça production, $46,3 \%$ of the consumers expressed a preference for artisan cachaças, $29,5 \%$ for industrial cachaças and $24,2 \%$ expressed not being able to distinguish the difference between the two types of cachaça.

Key words: Alcoholic beverage, cachaça consumption, artisan cachaça, industrial cachaça, consumption habits, consumer preferences
\end{abstract}

\footnotetext{
${ }^{1}$ Alunos do Programa de Pós-Graduação em Tecnologia Agroalimentar, do Centro de Ciências Humanas, Sociais e Agrárias, Campus Bananeiras, Universidade Federal da Paraíba, UFPB. E-mail: renanesoliveira@gmail.com; manosilva9001@hotmail.com

${ }^{2}$ Prof $^{a}$ do Dept ${ }^{0}$ de Gestão e Tecnologia Agroindústria, DGTA do Centro de Ciências Humanas, Sociais e Agrárias, Campus Bananeiras, UFPB. E-mail: edilma.coutinho@gmail.com

${ }^{3}$ Prof. do Dept ${ }^{\circ}$ de Engenharia de Alimentos do Centro de Tecnologia, Campus João Pessoa, UFPB. E-mail: ricardo.ufpb@gmail. com

${ }^{4}$ Prof. do Dept ${ }^{0}$ de Agropecuária do Centro de Ciências Humanas, Sociais e Agrárias, Campus Bananeiras, UFPB. E-mail: georgebeltrao@hotmail.com

* Autor para correspondência
} 


\section{Introdução}

A cachaça é uma bebida tipicamente brasileira, que vem conquistando espaço no mercado nacional e internacional. Segundo dados da IBRAC (2010), existem mais de 40 mil produtores de cachaça no país, que produzem aproximadamente 1,2 bilhões de litros da bebida anualmente. O setor de produção de cachaça é responsável pela geração de mais de 600 mil empregos diretos e indiretos.

Cerca de $75 \%$ da produção nacional de cachaça é realizada em empresas industriais, com produção em grande escala, que utilizam leveduras prensadas no processo de fermentação e destilam em colunas contínuas de aço inox. Por outro lado, a produção das cachaças denominadas de artesanais ou de alambique é de apenas 300 milhões de litros anuais e realizada em pequenas destilarias, que utilizam leveduras naturais na fermentação e destilam em alambiques de cobre, por permitir a separação das frações do destilado (PEREIRA, 2007; BARBOZA et al., 2010).

Jerônimo (2004) afirma que as empresas de cachaça estão buscando uma imagem fortemente vinculada à qualidade e investindo no desenvolvimento de um mercado consumidor mais exigente, fato que resulta no desenvolvimento de novos produtos, cujas características tentam desvinculá-la parcialmente de sua linha tradicional de bebida popular.

No Brasil, o setor de produção de cachaça vem desenvolvendo programas de qualidade para a bebida, com o objetivo de ampliar o mercado interno e melhorar as exportações. Entre os programas desenvolvidos, estão o Programa Brasileiro de Desenvolvimento da Cachaça (PBDAC) e o Programa Nacional de Certificação da Cachaça (PPNCC) (FERNANDES et al., 2005; ALBUQUERQUE et al., 2006; LOUREIRO, 2008; PRIBANIC, 2011).

De acordo com Vidal e Gonçalves (2008), no Nordeste, o setor produtivo de cachaça é caracterizado pela predominância de micro e pequenos produtores de baixo nível tecnológico. Coutinho (2001) registra que na região, o estado da Paraíba destaca-se como um dos principais produtores de cachaça de alambique, e uma das principais características do seu setor de produção é a coexistência de produtores arcaicos com modernos.

Desde 1993, mediante articulações e parcerias com diversos órgãos públicos e privados, o Governo do Estado da Paraíba vem implantando programas para o desenvolvimento e a consolidação da agroindústria da cachaça. Esse fato gerou resultados satisfatórios no processo e na gestão das empresas, que modernizaram suas unidades, desenvolveram novos produtos e novas marcas e ampliaram o mercado (EVARISTO; NÓBREGA, 2006; SILVA, 2011). Neste contexto inovador, até então, são desconhecidos trabalhos que identifiquem o perfil e os hábitos dos consumidores de cachaça, o que poderia gerar informações estratégicas para a conquista de novos mercados.

Segundo Matsunaga (2007), o panorama geral do mercado de alimentos é bastante competitivo e apresenta hoje uma grande variedade de produtos, por sua vez, os consumidores estão cada vez mais exigentes quanto aos atributos de qualidade dos produtos que consomem. Sob esse ponto de vista, é vital para a indústria processadora de cachaça, que almeja a conquista de novos mercados e busca desenvolver produtos diferenciados, conhecer o perfil, os hábitos e as preferências do consumidor.

O estudo do comportamento do consumidor em relação a alimentos e a bebidas tem caráter multidisciplinar, pois envolve várias áreas, tais como a ciência e tecnologia de alimentos, nutrição, psicologia e marketing. A percepção das características de um produto pelo consumidor pode ser influenciada por diversos fatores individuais que afetam a percepção dos atributos sensoriais, que interagem com fatores fisiológicos, comportamentais e cognitivos (NORONHA; DELIZA; SILVA, 2005).

Uma das formas de estudar o comportamento dos 
consumidores é prever o que eles provavelmente fariam sob determinadas condições. As previsões são fundamentadas em uma das três bases de informação: o que as pessoas dizem, o que fazem e o que fizeram, utilizando para essa identificação métodos como: pesquisa de intenção de compra, força de vendas e opiniões de especialistas (KOTLER; KELLER, 2006).

Diante do citado, neste trabalho, o objetivo foi estudar o perfil e os hábitos do consumidor paraibano de cachaça, avaliando a frequência de consumo, o tipo de cachaça preferido e o local de aquisição e de consumo da bebida.

\section{Metodologia}

\section{Coleta dos dados}

A pesquisa foi descritiva e quantitativa, realizada entre os meses de fevereiro a novembro de 2009. Foram estudados o perfil e os hábitos do consumidor de cachaça, por meio de questionários estruturados com nove perguntas fechadas e quatro abertas. Foram aplicados 595 questionários nas quatro mesorregiões paraibanas: 149 no Litoral, 149 no Agreste, 148 na Borborema e 149 no Sertão. Em cada mesorregião, foram selecionadas três cidades, onde se aplicaram entre 48 e 50 questionários. As questões fizeram referência a aspectos como gênero, estado civil, faixa etária, escolaridade, frequência de consumo, local de aquisição e de consumo da bebida e preferência entre a cachaça produzida de forma artesanal e industrial. Os entrevistadores foram treinados, e os entrevistados não foram identificados para assegurar a confidencialidade das informações. Participaram da pesquisa indivíduos que declararam serem consumidores de cachaça e não estarem alcoolizados.

\section{Análise dos dados}

Após a aplicação do questionário, foi editado um banco de dados contendo todas as informações para cada variável por cidade e mesorregião. Em seguida, foram tomadas as freqüências, por meio do procedimento freq do SAS (1996). A análise da frequência de consumo de cachaça foi realizada segundo as variáveis demográficas gênero, faixa etária e escolaridade, que foram consideradas variáveis independentes.

Foi realizado um teste de homogeneidade de variância pelo teste de razão máxima ou teste de Hartley, de acordo com o descrito por Banzato e Kronka (1989). Verificou-se que as variâncias não foram homogêneas, por esse motivo, os dados foram transformados em raiz quadrada $(\sqrt{x})$, por ser essa a forma mais adequada, em função de alguns valores serem iguais a zero.

O modelo matemático utilizado foi:

$$
\mathrm{Yij}=\mu+\mathrm{Mi}+\mathrm{eij}
$$

Em que:

$Y i j=$ variável testada;

$\mu=$ média comum às observações;

$\mathrm{Mi}=$ efeito da mesorregião (Litoral, Agreste, Borborema e Sertão);

eij $=$ efeito de fatores não controlados ou erro aleatório.

As médias foram comparadas pelo teste $\mathrm{t}$ de Student, a $5 \%$ de probabilidade, considerando os valores relativos ao coeficiente de variação e ao número de mesorregiões (tratamentos), de acordo com o apresentado por Sampaio (1998), utilizandose o procedimento GLM do SAS (1996). Para verificar a correlação entre os locais de aquisição e de consumo da cachaça, aplicou-se a analise de correlação de Pearson (r), determinado pelo procedimento CORR do SAS.

\section{Resultados e Discussão}

Participaram desta pesquisa 595 consumidores de cachaça na Paraíba, sendo 501 homens e 94 mulheres, o que corresponde a um percentual 
de $84,2 \%$ e $15,8 \%$, respectivamente, como é apresentado na Tabela 1. Na Paraíba, o estado civil predominante dos consumidores entrevistados foi solteiro $(54,8 \%)$, e os casados corresponderam a um percentual de 43,4\%. Diferentemente, na Mesorregião Litoral, observou-se que 51,7\% dos consumidores são casados, e 45,6\% são solteiros. A escolaridade dos consumidores de cachaça variou de educação pré-escolar incompleta a ensino superior completo. A maioria tem ensino médio completo $(28,1 \%)$. Cabe destacar que, na Mesorregião Sertão, quase todos os consumidores de cachaça cursaram o ensino fundamental incompleto $(23,5 \%)$, no entanto, apresenta o maior percentual de consumidores com apenas educação pré-escolar $(12,1 \%)$ e com ensino superior completo $(15,4 \%)$, em relação às demais mesorregiões.

Tabela 1. Dados demográficos dos consumidores de cachaça no Estado da Paraíba, 2010.

\begin{tabular}{|c|c|c|c|c|c|c|}
\hline & & & Mes & egiões* & & \\
\hline & s Demográficos & $\begin{array}{c}\text { Litoral } \\
\%\end{array}$ & $\begin{array}{c}\text { Agreste } \\
\%\end{array}$ & $\begin{array}{c}\text { Borborema } \\
\%\end{array}$ & $\begin{array}{c}\text { Sertão } \\
\%\end{array}$ & $\begin{array}{c}\text { Paraíba } \\
\%\end{array}$ \\
\hline Gênero & Masculino & 86,6 a & $89,3 \mathrm{a}$ & $75,0 \mathrm{a}$ & 85,9 a & 84,2 \\
\hline Genero & Feminino & $13,4 \mathrm{a}$ & $10,7 \mathrm{a}$ & $25,0 \mathrm{a}$ & $14,1 \mathrm{a}$ & 15,8 \\
\hline & Solteiro & $45,6 \mathrm{a}$ & $61,7 \mathrm{a}$ & $58,1 \mathrm{a}$ & $53,7 \mathrm{a}$ & 54,8 \\
\hline Estado civil & Casado & $51,7 \mathrm{a}$ & $38,3 \mathrm{a}$ & $41,2 \mathrm{a}$ & $42,3 \mathrm{a}$ & 43,4 \\
\hline & Outro & $2,7 \mathrm{a}$ & $0,0 \mathrm{a}$ & $0,7 \mathrm{a}$ & $4,0 \mathrm{a}$ & 1,8 \\
\hline & Pré-escolar incompleto & $6,0 \mathrm{a}$ & $9,4 \mathrm{a}$ & $5,4 \mathrm{a}$ & $12,1 \mathrm{a}$ & 8,2 \\
\hline & Pré-escolar completo & $1,3 \mathrm{a}$ & $0,7 \mathrm{a}$ & $1,4 \mathrm{a}$ & $4,0 \mathrm{a}$ & 1,8 \\
\hline & Fund. Incompleto & $12,4 \mathrm{a}$ & $28,2 \mathrm{a}$ & $16,2 \mathrm{a}$ & $23,5 \mathrm{a}$ & 20,8 \\
\hline Fscolaridade & Fundamental completo & $10,0 \mathrm{a}$ & $8,1 \mathrm{a}$ & $4,7 \mathrm{a}$ & $7,4 \mathrm{a}$ & 7,5 \\
\hline Escoraridade & Médio Incompleto & $19,5 \mathrm{a}$ & $10,1 \mathrm{a}$ & $14,2 \mathrm{a}$ & $10,7 \mathrm{a}$ & 13,6 \\
\hline & Médio Completo & $36,2 \mathrm{a}$ & $25,5 \mathrm{ab}$ & $29,7 \mathrm{ab}$ & $20,8 \mathrm{a}$ & 28,1 \\
\hline & Superior Incompleto & $8,1 \mathrm{a}$ & $10,7 \mathrm{a}$ & $19,6 \mathrm{a}$ & $6,0 \mathrm{a}$ & 11,1 \\
\hline & Superior completo & $6,0 \mathrm{a}$ & $7,4 \mathrm{a}$ & $8,8 \mathrm{a}$ & $15,4 \mathrm{a}$ & 9,4 \\
\hline & $<18$ & $2,7 \mathrm{a}$ & $1,3 \mathrm{a}$ & $4,7 \mathrm{a}$ & $2,0 \mathrm{a}$ & 2,7 \\
\hline & $18-25$ & $17,4 \mathrm{a}$ & $35,6 \mathrm{a}$ & $35,1 \mathrm{a}$ & $25,5 \mathrm{a}$ & 28,4 \\
\hline Faixa etária & $26-35$ & $29,5 \mathrm{ab}$ & $37,6 \mathrm{a}$ & $20,3 \mathrm{~b}$ & $34,2 \mathrm{ab}$ & 30,4 \\
\hline & $36-45$ & $27,5 \mathrm{a}$ & $12,7 \mathrm{a}$ & $22,3 \mathrm{a}$ & $17,5 \mathrm{a}$ & 20,0 \\
\hline & $>45$ & $22,8 \mathrm{a}$ & $12,8 \mathrm{a}$ & $17,6 \mathrm{a}$ & $20,8 \mathrm{a}$ & 18,5 \\
\hline
\end{tabular}

*Letras diferentes na mesma linha diferem estatisticamente $(\mathrm{P}<0,05)$ entre si pelo teste $\mathrm{t}$.

$\mathrm{Na}$ Paraíba, 28,4\% dos entrevistados tinham entre 18 e 25 anos, e $30,4 \%$, entre 26 e 35 anos. O maior consumo entre 18 e 25 anos foi observado no Agreste (35,6\%) e na Borborema (35,1\%). Nessa mesorregião, também foi observado o maior consumo entre os menores de 18 anos $(4,7 \%)$.

Vieira e Pereira (2006) avaliaram o perfil do consumidor de vinho em bares noturnos, no município de Cascavel/PR, e registraram que 31,4\% da amostra encontravam-se entre 18 e 23 anos. A partir desse fato, os autores comentaram que os indivíduos estão começando a consumir bebidas alcoólicas cada vez mais cedo. Percepção similar foi argumentada por Balan e Campos (2006), que observaram que $23,8 \%$ das graduandas do Curso de Enfermagem de Campinas/SP experimentaram bebidas alcoólicas pela primeira vez entre 15 e 18 anos. Strauch et al. (2009) estudaram o uso de álcool por adolescentes e identificaram o consumo na faixa etária entre 11 e 15 anos. Os autores salientam o inicio extremamente precose do uso de bebidas alcoólicas. 
Nas quatro mesorregiões, observou-se uma diferença significativa em relação à escolaridade no ensino médio completo e à faixa etária de 26 a 35 anos. A Mesorregião Litoral apresentou o maior percentual de consumidores com nível de escolaridade médio completo, o que diferiu significativamente da Mesorregião Sertão. No entanto, essas mesorregiões não se diferenciaram da Mesorregião Agreste e da Borborema, que se diferenciaram significativamente na faixa etária de 26 a 35 anos.

A frequência de consumo por mesorregião está retratada na Tabela 2, onde se pode observar que o consumo predominante foi semanal nas quatro mesorregiões. Na Paraíba, a frequência diária foi $13,9 \%$, a semanal, 44,4\%, a mensal, $12,4 \%$, e os que responderam raramente foi $17,8 \%$. O maior consumo diário foi observado no Litoral $(19,5 \%)$, e o menor, na Borborema $(8,8 \%)$. É importante salientar que a Mesorregião Sertão teve a menor frequência de consumo quinzenal $(8,7 \%)$ e a maior raramente $(23,5 \%)$. Não houve diferença significativa $(\mathrm{P}<0,05)$ entre as mesorregiões em relação à sua frequência de consumo.

Tabela 2. Distribuição percentual da freqüência de consumo de cachaça nas mesorregiões do Estado da Paraíba, 2010.

\begin{tabular}{|c|c|c|c|c|c|}
\hline \multirow[b]{2}{*}{ Freqüência de consumo } & \multicolumn{4}{|c|}{ Mesorregiões* } & \multirow[b]{2}{*}{$\begin{array}{c}\text { Paraíba } \\
\%\end{array}$} \\
\hline & $\begin{array}{c}\text { Litoral } \\
\%\end{array}$ & $\begin{array}{c}\text { Agreste } \\
\% \\
\end{array}$ & $\begin{array}{c}\text { Borborema } \\
\%\end{array}$ & $\begin{array}{c}\text { Sertão } \\
\%\end{array}$ & \\
\hline Diária & $19,5 \mathrm{a}$ & $13,4 \mathrm{a}$ & $8,8 \mathrm{a}$ & $14,1 \mathrm{a}$ & 13,9 \\
\hline Semanal & $43,6 \mathrm{a}$ & $38,9 \mathrm{a}$ & $53,4 \mathrm{a}$ & $41,6 \mathrm{a}$ & 44,4 \\
\hline Quinzenal & $10,1 \mathrm{a}$ & $17,5 \mathrm{a}$ & $13,5 \mathrm{a}$ & $8,7 \mathrm{a}$ & 12,4 \\
\hline Mensal & $8,1 \mathrm{a}$ & $13,4 \mathrm{a}$ & $12,2 \mathrm{a}$ & $12,1 \mathrm{a}$ & 11,4 \\
\hline Rara & $18,8 \mathrm{a}$ & $16,8 \mathrm{a}$ & $12,2 \mathrm{a}$ & $23,5 \mathrm{a}$ & 17,8 \\
\hline Total & 100,0 & 100,0 & 100,0 & 100,0 & 100,0 \\
\hline
\end{tabular}

*Letras diferentes na mesma linha diferem estatisticamente $(\mathrm{P}<0,05)$ entre si pelo teste $\mathrm{t}$.

Nos estudos de Carneiro (2007) sobre marca de cachaça, foi observado um consumo semanal da bebida em $40 \%$ dos entrevistados, valor próximo ao encontrado nesta pesquisa. Um fator importante a ser considerado é que o consumo diário de bebida alcoólica é um risco para a saúde. Com essa visão, autores como Barros et al. (2008) e Mascarenhas et al. (2009), que estudaram o consumo de bebidas alcoólicas sob o ponto de vista da saúde pública, alertam que o uso abusivo de álcool deve ser evitado.

$\mathrm{Na}$ Tabela 3, estão apresentadas as frequencias de consumo de cachaça nas quatro mesorregões paraibanas, segundo as variáveis demograficas gênero, grau de instrução e faixa etária.

Entre os homens, a maior frequência de consumo foi semanal em todas as mesorregiões. Por sua vez, o público feminino revelou uma frequência menor de consumo de cachaça. Esses dados estão de acordo com as pesquisas de Laranjeira et al. (2007), que realizaram um levantamento sobre os padrões de consumo de álcool na população brasileira e observaram que os homens apresentam porcentagem mais alta do que as mulheres no que diz respeito ao alto consumo diário e semanal. 
Tabela 3. Distribuição percentual da frequência de consumo de cachaça de acordo com as variáveis demográficas (gênero, escolaridade e faixa etária), nas mesorregiões do Estado da Paraíba, 2010.

\begin{tabular}{|c|c|c|c|c|c|c|c|c|}
\hline \multirow{4}{*}{ Frequência de consumo } & \multicolumn{8}{|c|}{ Gênero ${ }^{1}$ (Mesorregiões) } \\
\hline & \multicolumn{2}{|c|}{ Litoral } & \multicolumn{2}{|c|}{ Agreste } & \multicolumn{2}{|c|}{ Borborema } & \multicolumn{2}{|c|}{ Sertão } \\
\hline & $\mathrm{M}$ & $\mathrm{F}$ & M & $\mathrm{F}$ & M & $\mathrm{F}$ & M & $\mathrm{F}$ \\
\hline & $\%$ & $\%$ & $\%$ & $\%$ & $\%$ & $\%$ & $\%$ & $\%$ \\
\hline Diária & $21,7^{\mathrm{a}}$ & $5,0^{\mathrm{b}}$ & $15,0^{\mathrm{a}}$ & $0,0^{\mathrm{b}}$ & $11,7^{\mathrm{a}}$ & $0,0^{\mathrm{b}}$ & $15,6^{\mathrm{a}}$ & $4,8^{\mathrm{b}}$ \\
\hline Semanal & $44,9^{\mathrm{a}}$ & $35,0^{\mathrm{b}}$ & $42,9^{\mathrm{a}}$ & $6,3^{\mathrm{b}}$ & $54,9^{\mathrm{a}}$ & $48,6^{\mathrm{b}}$ & $44,5^{\mathrm{a}}$ & $23,8^{b}$ \\
\hline Quinzenal & $10,1^{\mathrm{a}}$ & $10,0^{\mathrm{b}}$ & $17,3^{\mathrm{a}}$ & $18,7^{\mathrm{b}}$ & $10,8^{\mathrm{a}}$ & $21,6^{\mathrm{b}}$ & $8,6^{\mathrm{a}}$ & $9,5^{\mathrm{b}}$ \\
\hline Mensal & $8,5^{\mathrm{a}}$ & $5,0^{\mathrm{b}}$ & $12,8^{\mathrm{a}}$ & $18,7^{\mathrm{b}}$ & $9,9^{\mathrm{a}}$ & $18,9^{\mathrm{b}}$ & $11,7^{\mathrm{a}}$ & $14,3^{\mathrm{b}}$ \\
\hline Rara & $14,7^{\mathrm{a}}$ & $45,0^{\mathrm{b}}$ & $12,0^{\mathrm{a}}$ & $56,3^{\mathrm{b}}$ & $12,6^{\mathrm{a}}$ & $10,8^{\mathrm{b}}$ & $19,5^{\mathrm{a}}$ & $47,6^{\mathrm{b}}$ \\
\hline Total & 100,0 & 100,0 & 100,0 & 100,0 & 100,0 & 100,0 & 100,0 & 100,0 \\
\hline
\end{tabular}

\begin{tabular}{|c|c|c|c|c|c|c|c|c|c|c|c|c|c|c|c|c|}
\hline \multirow{4}{*}{ Freq. } & \multicolumn{16}{|c|}{ Escolaridade $^{2}$ (Mesorregiões) } \\
\hline & \multicolumn{4}{|c|}{ Litoral } & \multicolumn{4}{|c|}{ Agreste } & \multicolumn{4}{|c|}{ Borborema } & \multicolumn{4}{|c|}{ Sertão } \\
\hline & $\mathrm{P}$ & $\mathrm{F}$ & M & $\mathrm{S}$ & $\mathrm{P}$ & $\mathrm{F}$ & M & $\mathrm{S}$ & $\mathrm{P}$ & $\mathrm{F}$ & M & $\mathrm{S}$ & $\mathrm{P}$ & $\mathrm{F}$ & M & $\mathrm{S}$ \\
\hline & $\%$ & $\%$ & $\%$ & $\%$ & $\%$ & $\%$ & $\%$ & $\%$ & $\%$ & $\%$ & $\%$ & $\%$ & $\%$ & $\%$ & $\%$ & $\%$ \\
\hline Dia. $^{3}$ & $45,5^{\mathrm{c}}$ & $20,6^{\mathrm{b}}$ & $19,3^{\mathrm{a}}$ & $4,8^{\mathrm{d}}$ & $26,7^{\mathrm{c}}$ & $14,8^{\mathrm{a}}$ & $11,3^{\mathrm{b}}$ & $7,4^{\mathrm{d}}$ & $10,0^{\mathrm{d}}$ & $19,4^{\mathrm{a}}$ & $6,2^{\mathrm{b}}$ & $4,8^{c}$ & $25,0^{\mathrm{b}}$ & $21,7^{\mathrm{a}}$ & $6,4^{c}$ & $6,3^{\mathrm{d}}$ \\
\hline Sem. & $45,5^{\mathrm{d}}$ & $47,1^{\mathrm{b}}$ & $41,0^{\mathrm{a}}$ & $47,6^{\mathrm{c}}$ & $46,7^{\mathrm{d}}$ & $46,3^{\mathrm{a}}$ & $28,3^{\mathrm{b}}$ & $40,7^{\mathrm{c}}$ & $70,0^{\mathrm{d}}$ & $54,8^{\mathrm{c}}$ & $50,8^{\mathrm{a}}$ & $52,4^{\mathrm{b}}$ & $41,7^{\mathrm{c}}$ & $43,5^{b}$ & $55,3^{\mathrm{a}}$ & $18,8^{\mathrm{d}}$ \\
\hline Quin. & $9,1^{\mathrm{d}}$ & $8,8^{\mathrm{b}}$ & $10,8^{\mathrm{a}}$ & $9,5^{\mathrm{c}}$ & $6,7^{\mathrm{d}}$ & $20,4^{\mathrm{a}}$ & $15,1^{\mathrm{b}}$ & $22,2^{\mathrm{c}}$ & $10,0^{\mathrm{d}}$ & $9,7^{\mathrm{c}}$ & $13,8^{\mathrm{b}}$ & $16,7^{\mathrm{a}}$ & $8,3^{\mathrm{c}}$ & $0,0^{\mathrm{d}}$ & $14,9^{\mathrm{a}}$ & $12,5 b$ \\
\hline Mens & $0,0^{\mathrm{d}}$ & $5,9^{c}$ & $7,2^{\mathrm{a}}$ & $19,0^{\mathrm{b}}$ & $0,0^{\mathrm{d}}$ & $13,0^{\mathrm{b}}$ & $18,9^{\mathrm{a}}$ & $11,1^{\mathrm{c}}$ & $0,0^{\mathrm{d}}$ & $9,7^{\mathrm{c}}$ & $12,3^{\mathrm{a}}$ & $16,7^{\mathrm{b}}$ & $16,7^{\mathrm{b}}$ & $6,5^{\mathrm{a}}$ & $4,3^{\mathrm{d}}$ & $28,1^{\mathrm{a}}$ \\
\hline Raro & $0,0^{\mathrm{d}}$ & $17,6^{\mathrm{b}}$ & $21,7^{\mathrm{a}}$ & $19,0^{\mathrm{c}}$ & $20,0^{c}$ & $5,6^{\mathrm{c}}$ & $26,4^{\mathrm{a}}$ & $18,5^{\mathrm{b}}$ & $10,0^{\mathrm{d}}$ & $6,5^{\mathrm{c}}$ & $16,9^{b}$ & $9,5^{\mathrm{a}}$ & $8,3^{\mathrm{d}}$ & $28,3^{c}$ & $19,1^{\mathrm{c}}$ & $34,4^{b}$ \\
\hline Total & 100 & 100 & 100 & 100 & 100 & 100 & 100 & 100 & 100 & 100 & 100 & 100 & 100 & 100 & 100 & 100 \\
\hline
\end{tabular}

\begin{tabular}{|c|c|c|c|c|c|c|c|c|c|c|c|c|}
\hline \multirow{3}{*}{ Freq. } & \multicolumn{12}{|c|}{ Faixa etária (Mesorregiões) } \\
\hline & \multicolumn{3}{|c|}{ Litoral } & \multicolumn{3}{|c|}{ Agreste } & \multicolumn{3}{|c|}{ Borborema } & \multicolumn{3}{|c|}{ Sertão } \\
\hline & $\begin{array}{c}\text { Ate } 25 \\
\%\end{array}$ & $\begin{array}{c}26-35 \\
\%\end{array}$ & $\begin{array}{c}>35 \\
\%\end{array}$ & $\begin{array}{c}\text { Ate } 25 \\
\%\end{array}$ & $\begin{array}{c}26-35 \\
\%\end{array}$ & $\begin{array}{c}>35 \\
\%\end{array}$ & $\begin{array}{c}\text { Ate } 25 \\
\%\end{array}$ & $\begin{array}{c}26-35 \\
\%\end{array}$ & $\begin{array}{c}>35 \\
\%\end{array}$ & $\begin{array}{c}\text { Ate } 25 \\
\%\end{array}$ & $\begin{array}{c}26-35 \\
\%\end{array}$ & $\begin{array}{c}>35 \\
\%\end{array}$ \\
\hline Diario & $8,9^{c}$ & $9,1^{\mathrm{b}}$ & $29,3^{\mathrm{a}}$ & $5,5^{\mathrm{c}}$ & $12,5^{\mathrm{b}}$ & $26,3^{\mathrm{a}}$ & $3,4^{c}$ & $13,3^{\mathrm{b}}$ & $11,9^{\mathrm{a}}$ & $14,6^{\mathrm{c}}$ & $13,7^{\mathrm{b}}$ & $14,0^{\mathrm{a}}$ \\
\hline Semanal & $28,9^{\mathrm{c}}$ & $36,4^{\mathrm{b}}$ & $50,7^{\mathrm{a}}$ & $29,1^{\mathrm{c}}$ & $42,9^{\mathrm{a}}$ & $47,4^{\mathrm{b}}$ & $57,6^{\mathrm{a}}$ & $46,7^{\mathrm{c}}$ & $52,5^{\mathrm{b}}$ & $36,6^{\mathrm{c}}$ & $43,1^{\mathrm{b}}$ & $43,9^{\mathrm{a}}$ \\
\hline Quinzenal & $20,0^{\mathrm{a}}$ & $11,4^{\mathrm{b}}$ & $5,3^{\mathrm{c}}$ & $21,8^{\mathrm{a}}$ & $16,1^{\mathrm{b}}$ & $13,2^{\mathrm{c}}$ & $16,9^{\mathrm{a}}$ & $3,3^{\mathrm{c}}$ & $15,3^{\mathrm{b}}$ & $17,1^{\mathrm{a}}$ & $9,8^{\mathrm{b}}$ & $1,8^{\mathrm{c}}$ \\
\hline Mensal & $17,8^{\mathrm{b}}$ & $11,4^{\mathrm{a}}$ & $4,0^{\mathrm{c}}$ & $16,4^{\mathrm{a}}$ & $14,3^{\mathrm{b}}$ & $7,9^{\mathrm{c}}$ & $11,9^{\mathrm{a}}$ & $23,3^{\mathrm{a}}$ & $6,8^{\mathrm{b}}$ & $9,8^{\mathrm{b}}$ & $13,7^{\mathrm{a}}$ & $12,3^{\mathrm{a}}$ \\
\hline Raro & $24,4^{\mathrm{c}}$ & $31,8^{\mathrm{a}}$ & $10,7^{\mathrm{b}}$ & $27,3^{\mathrm{a}}$ & $14,3^{\mathrm{b}}$ & $5,3^{\mathrm{c}}$ & $10,2^{\mathrm{b}}$ & $13,3^{\mathrm{c}}$ & $13,6^{\mathrm{a}}$ & $22,0^{c}$ & $19,6^{\mathrm{b}}$ & $28,1^{\mathrm{a}}$ \\
\hline Total & 100,0 & 100,0 & 100,0 & 100,0 & 100,0 & 100,0 & 100,0 & 100,0 & 100,0 & 100,0 & 100,0 & 100,0 \\
\hline
\end{tabular}

${ }^{\mathrm{M}} \mathrm{M}$ - Masculino; F - Feminino;

${ }^{2} \mathrm{P}$ - Pré-escolar; F - Fundamental; M - Médio; S - Superior;

${ }^{3}$ Dia - diariamente; Sem - semanalmente; Quin - quinzenalmente; Mens - mensalmente;

*Letras diferentes na mesma linha, dentro de cada mesorregião para cada freqüência de consumo, diferem estatisticamente $(\mathrm{P}<0,05)$ entre si pelo teste t.

Convém registrar a frequência predominante de (56,3\%) e no Sertão (47,6\%). Na Mesorregião consumo de cachaça dos homens por mesorregião: Borborema, a frequência predominante do consumo no Litoral, o consumo semanal é de 44,9\%, e diário, de cachaça das mulheres foi semanal (48,6\%). Não de 21,7\%; no Agreste, a semanal é de 42,9\%, e foi observado o consumo diário de cachaça entre quinzenal, de 17,3\%; em Borborema, a semanal as mulheres nas mesorregiões do Agreste e da é de $54,9 \%$, e rara, 12,6\%; no Sertão, o consumo Borborema. semanal é de $44,5 \%$, e rara, $19,5 \%$.

A frequência de consumo predominate das

No estudo da frequência de consumo, segundo mulheres foi rara no Litoral $(45,0 \%)$, no Agreste a variável escolaridade, pode-se observar que o consumo diário e semanal foi maior entre os 
consumidores de baixa escolaridade, e os com ensino superior apresentaram o menor consumo diário. A soma das percentagens da frequência diária e semanal no nível infantil de escolaridade foi 91,0\% no Litoral, 73,4\% em Boborema, 80,0\% no Agreste e 66,7\% no Sertão. Em relação ao nível de escolaridade Superior, a soma das percentagens da frequência diária e semanal foi $52,4 \%$ no Litoral, $48,1 \%$ em Boborema, 57,2\% no Agreste e 25,1\% no Sertão.

Barros et al. (2008) registraram que a literatura é contraditória quanto ao consumo de bebidas alcoólicas nos segmentos socais. Ainda assim, os autores salietaram que existe a tendência de afirmar maior consumo nos estratos sociais de menos renda e escolaridade.

Em todas as faixas etárias, a frequência de consumo de cachaça identificada foi predominantemente semanal. Exceto no Sertão, o menor consumo diário foi observado entre os jovens com até 25 anos. $\mathrm{O}$ consumo diário foi mais intenso entre os consumidores acima de 35 anos, no Litoral (29,3\%), e no Agreste (26,3\%).

Para Laranjeira et al. (2007), a frequência de consumo varia muito, no que diz respeito às faixas etárias. Os autores identificaram que a frequência em relação a beber pelo menos uma vez por semana é mais intensa na faixa etária entre 26 e 35 anos.
Em relação à variável demográfica gênero, os homens se diferenciaram estatisticamente das mulheres em todas as mesorregiões e em todas as frequências de consumo. Em relação à escolaridade, houve diferença estatística em todas as mesorregões e frequências. O mesmo aconteceu com a variável demográfica faixa estária. Ou seja, houve diferença estatística significativa $(\mathrm{p}<0,05)$ para todas as variáveis avaliadas dentro das mesorregiões e por frequência de consumo.

$\mathrm{Na}$ Tabela 4, é interessante observar que em todas as mesorregiões a aquisição da cachaça é realizada majoritariamente em bares, seguida de supermercados. A compra de cachaça em depositos de bebidas foi identificada apenas no Litoral, onde 7,4\% dos entrevistados adquirem a cachaça nesse canal de comercialização. A compra da cachaça direto do produtor foi relevante no Agreste (4,7\%), onde municípios como Guarabira, Bananeiras, Borborema e Areia têm alguns engenhos de cachaça produzida artesanalmente, com ponto de venda para o consumidor, especialmente os engenhos que não são legalizados.

A opção por comprar em restaurantes apresentou diferenças significativas entre as mesorregiões. A mesorregião Litoral diferiu significativamente da mesorregião Borborema $(p<0,05)$, no que diz respeito ao local de aquisição em restaurantes.

Tabela 4. Local de aquisição de cachaça nas mesorregiões do Estado da Paraíba, 2010.

\begin{tabular}{lccccc}
\hline & \multicolumn{4}{c}{ Mesorregião } & Paraíba \\
\cline { 2 - 4 } \multicolumn{1}{c}{ Local de aquisição } & Litoral & Agreste & Borborema & Sertão & \% \\
& $\%$ & $\%$ & $\%$ & $\%$ & $\%$ \\
\hline Supermercados & $23,5 \mathrm{a}$ & $28,2 \mathrm{a}$ & $23,6 \mathrm{a}$ & $29,5 \mathrm{a}$ & 26,2 \\
Bares & $46,3 \mathrm{a}$ & $49,7 \mathrm{a}$ & $52,7 \mathrm{a}$ & $57,1 \mathrm{a}$ & 51,4 \\
Restaurantes & $0,7 \mathrm{ab}$ & $2,7 \mathrm{ab}$ & $0,0 \mathrm{a}$ & $3,4 \mathrm{~b}$ & 1,7 \\
Direto do produtor & $0,0 \mathrm{a}$ & $4,7 \mathrm{a}$ & $0,7 \mathrm{a}$ & $1,3 \mathrm{a}$ & 1,7 \\
Supermercados ou bares & $11,4 \mathrm{a}$ & $4,0 \mathrm{a}$ & $16,1 \mathrm{a}$ & $6,7 \mathrm{a}$ & 9,6 \\
Depósito de bebidas & $7,4 \mathrm{a}$ & $0,0 \mathrm{~b}$ & $0,0 \mathrm{~b}$ & $0,0 \mathrm{~b}$ & 1,8 \\
Bares ou restaurantes & $0,7 \mathrm{a}$ & $1,3 \mathrm{a}$ & $1,4 \mathrm{a}$ & $0,7 \mathrm{a}$ & 1,0 \\
Supermercados/bares/restaurante & $0,0 \mathrm{a}$ & $1,3 \mathrm{a}$ & $0,7 \mathrm{a}$ & $1,3 \mathrm{a}$ & 0,8 \\
Outros & $10,1 \mathrm{a}$ & $8,1 \mathrm{ab}$ & $4,1 \mathrm{~b}$ & $0,0 \mathrm{c}$ & 5,6 \\
Total & 100,0 & 100,0 & 100,0 & 100,0 & 100,0 \\
\hline
\end{tabular}

*Letras diferentes na mesma linha diferem estatisticamente $(\mathrm{P}<0,05)$ entre si pelo teste $\mathrm{t}$. 
Conforme se pode observar na Tabela 5, 24,3\%, em casa, em bares ou em restaurantes, na Paraíba, 45,2\% dos consumidores preferem e 20,7\%, apenas em casa. O consumo em festas consumir a cachaça em bares ou restaurantes, variou de 2,7\%, em Borborema, a 0,7\%, no Sertão.

Tabela 5. Local de consumo de cachaça nas mesorregiões do Estado da Paraíba, 2010.

\begin{tabular}{lccccc}
\hline \multirow{2}{*}{ Local de consumo } & \multicolumn{4}{c}{ Mesorregião } \\
\cline { 2 - 5 } & $\begin{array}{c}\text { Litoral } \\
\%\end{array}$ & $\begin{array}{c}\text { Agreste } \\
\%\end{array}$ & $\begin{array}{c}\text { Borborema } \\
\%\end{array}$ & $\begin{array}{c}\text { Sertão } \\
\%\end{array}$ & $\begin{array}{c}\text { Paraíba } \\
\%\end{array}$ \\
\hline Casa & $26,9 \mathrm{a}$ & $20,8 \mathrm{a}$ & $15,5 \mathrm{a}$ & $19,5 \mathrm{a}$ & 20,7 \\
Bares ou restaurante & $48,3 \mathrm{a}$ & $44,3 \mathrm{a}$ & $43,9 \mathrm{a}$ & $44,3 \mathrm{a}$ & 45,2 \\
Casa, bares ou restaurante & $14,8 \mathrm{a}$ & $22,2 \mathrm{ab}$ & $35,1 \mathrm{~b}$ & $24,2 \mathrm{ab}$ & 24,0 \\
Festas & $2,0 \mathrm{a}$ & $2,7 \mathrm{a}$ & $2,7 \mathrm{a}$ & $0,6 \mathrm{a}$ & 2,0 \\
Todos os lugares & $2,7 \mathrm{a}$ & $2,0 \mathrm{a}$ & $0,0 \mathrm{a}$ & $0,7 \mathrm{a}$ & 1,3 \\
Casas noturnas & $0,0 \mathrm{a}$ & $0,7 \mathrm{a}$ & $2,0 \mathrm{a}$ & $1,3 \mathrm{a}$ & 1,0 \\
Outros & $5,4 \mathrm{a}$ & $7,4 \mathrm{a}$ & $0,7 \mathrm{a}$ & $9,4 \mathrm{a}$ & 5,7 \\
Total & 100,0 & 100,0 & 100,0 & 100,0 & 100,0 \\
\hline
\end{tabular}

*Letras diferentes na mesma linha diferem estatisticamente $(\mathrm{P}<0,05)$ entre si pelo teste $\mathrm{t}$.

Os resultados desta pesquisa diferem dos dados encontrados na bibliografia estudada. Carneiro (2007) verificou que, na cidade de Viçosa/MG, $72,0 \%$ dos consumidores preferiam consumir cachaça em bares ou casas noturnas. Costa e Cavalcante (2007) estudaram o comportamento do consumidor de cerveja e observaram que 3,82\% preferiam consumir a cerveja em clubes, festas, bares e restaurantes. Reis (2008) verificou que o consumo de bebida alcoólica é mais intenso nas festas em $76,6 \%$ dos alunos do ensino fundamental e do médio.

As mesorregiões Litoral e Borborema apresentaram diferença significativa entre si, no nível de 5\% $(\mathrm{P}<0,05)$, em relação ao consumo em casa, em bares ou em restaurantes. O Agreste e o Sertão não diferem das demais regiões.

A correlação entre os locais de aquisição e de consumo da cachaça foi realizada por meio da análise de Pearson (r). Segundo Figueiredo Filho e Silva Junior (2009), o coeficiente de correlação de Pearson é uma medida do grau de relação linear entre duas variáveis quantitativas e seus valores variam entre -1 e 1 . O valor 0 (zero) significa que não há relação linear, o valor 1 indica uma relação linear perfeita e o valor -1 também indica uma relação linear perfeita mas inversa, ou seja, quando uma das variáveis aumenta a outra diminui.

Na Tabela 6 está apresentada a correlação entre os locais de aquisição e de consumo da cachaça. Verifica-se que quando o consumo de cachaça é realizado em casa, a bebida tende a ser adquirida em depósitos $(0,64)$ e supermercados $(0,49)$. Também houve uma correlação positiva entre o consumo de cachaça em festas e a aquisição em bares e restaurantes $(0,73)$. Para as cachaças consumidas em casas noturnas, existe a tendência de aquisição das cachaças em supermercados e bares $(0,57)$.

A Tabela 7 descreve a preferência dos consumidores paraibanos entre a cachaça produzida artesanalmente e a produzida industrialmente. No estado, 50,4\% dos consumidores relataram que preferem as cachaças de produção industrial; $31,8 \%$ preferem as cachaças de produção artesanal, e $17,8 \%$ não distinguem os dois tipos de cachaça. O Litoral (70,5\%) e o Sertão $(65,1 \%)$ apresentaram os maiores percentuais de preferência pela industrial. $\mathrm{Na}$ Borborema, foi observado que 38,5\% dos 
consumidores não sabem a diferença entre a cachaça de produção artesanal e a de produção industrial. No Agreste, reduto paraibano que produz cachaça de forma artesanal, 46,3\% relataram prefeir cachaça de produção artesanal, 29,5\%, de produção industrial, e $24,2 \%$ não distinguem a diferença.
A preferência de consumo entre as cachaças de processo artesanal e industrial não apresentou diferença estatística entre as mesorregiões, com excessão do consumo de cachaça artesanal no Agreste.

Tabela 6. Coeficiente de correlação entre o local de aquisição e o local de consumo da cachaça, 2010.

\begin{tabular}{lcccccc}
\hline \multirow{2}{*}{ Local de aquisição } & \multicolumn{7}{c}{ Local de consumo } \\
\cline { 2 - 6 } & Casa & Bares/Rest & C/B/R & Festas & Casa Noturna & Td. Lugar $^{* *}$ \\
\hline Supermercados & 0,49 & $-0,38$ & $-0,08$ & 0,01 & 0,51 & 0,34 \\
Bares & $-0,72$ & 0,51 & 0,15 & 0,01 & $-0,48$ & $-0,27$ \\
Restaurantes & $-0,02$ & $-0,17$ & $-0,04$ & 0,44 & 0,27 & 0,53 \\
Sup. ou bares & 0,31 & $-0,33$ & 0,23 & $-0,22$ & 0,57 & $-0,33$ \\
Bares ou restaurantes & $-0,16$ & $-0,31$ & 0,34 & 0,73 & $-0,34$ & 0,41 \\
Direto do produtor & $-0,08$ & $-0,10$ & 0,02 & $-0,43$ & $-0,23$ & $-0,42$ \\
Depósito & 0,64 & $-0,17$ & $-0,45$ & 0,00 & $-0,33$ & 0,32 \\
\hline
\end{tabular}

*Casa, bares e restaurantes; ** Todos os lugares.

Tabela 7. Preferência entre cachaça produzida de forma artesanal e industrial nas mesorregiões do Estado da Paraíba, 2010.

\begin{tabular}{lccccc}
\hline \multirow{2}{*}{ Tipo de cachaça preferido } & \multicolumn{2}{c}{ Mesorregiões* } \\
& Litoral & Agreste & Borborema & Sertão & Paraíba \\
$\%$ & $\%$ & $\%$ & $36,5 \mathrm{a}$ & $65,1 \mathrm{a}$ & 50,4 \\
\hline Industrial & $70,5 \mathrm{a}$ & $29,5 \mathrm{a}$ & $25,0 \mathrm{a}$ & $28,9 \mathrm{a}$ & 31,8 \\
Artesanal & $26,8 \mathrm{a}$ & $46,3 \mathrm{~b}$ & $38,5 \mathrm{a}$ & $6,0 \mathrm{a}$ & 17,8 \\
Não distingui & $2,7 \mathrm{a}$ & $24,2 \mathrm{a}$ & 100,0 & 100,0 & 100,0 \\
Total & 100,0 & 100,0 & 100 & \\
\hline
\end{tabular}

*Letras diferentes na mesma linha diferem estatisticamente $(\mathrm{P}<0,05)$ entre si pelo teste $\mathrm{t}$.

\section{Conclusão}

No estado da Paraíba, a maioria dos consumidores de cachaça é masculina, com idade variando entre dezoito e trinta e cinco anos, e ensimo médio completo. Em todas as mesorregiões da Paraíba, a maior frequência de consumo de cachaça foi semanal. Nesse aspecto, as mulheres revelaram uma frequência de consumo menor que os homens. A frequência de consumo diário e semanal foi maior entre os consumidores de baixa escolaridade. Os consumidores com ensino superior apresentaram o menor consumo diário. Os locais preferidos para adquirir a cachaça foram os bares e os supermercados. Muitos entrevistados revelaram que consomem a cachaça em casa. Quando o consumo da cachaça é realizado em festas, existe a tendência de a bebida ser adquirida em bares e restaurantes. A cachaça produzida industrialmenete foi apontada como a preferida pelos consumidores paraibanos, entretanto, na Mesorregião do Agreste, reduto que produz a cachaça de forma artesanal, os consumidores relataram preferência por cachaça produzida artesanalmenete. Em todo o estado, um percentual elevado de consumidores relatou que não sabe a diferença entre cachaça de produção artesanal e industrial. 


\section{Procedimento Ético}

A pesquisa foi aprovada pelo Comitê de Ética em Pesquisa, do Hospital Lauro Wanderley, da Universidade Federal da Paraíba, sob o protocolo no $455 / 10$, folha de rosto $\mathrm{n}^{\circ} 363100$.

\section{Referências}

ALBUQUERQUE, G. J. K.; SAMPAIO, C. M. S.; TINELLI, F. M.; RIBEIRO, L. P.; PINTO, P. S. Programas de avaliação da conformidade desenvolvidos pelo Inmetro na área agropecuária. In: CONFERÊNCIA INTERNACIONAL SOBRE RASTREABILIDADE DE PRODUTOS AGROPECUÁRIOS, 2., 2006. Anais... Brasília: Ministério da Agricultura, Pecuária e Abastecimento, 2006.

BALAN, T. G.; CAMPOS, C. J. G. Padrão do consumo de bebidas alcoólicas entre graduandas de enfermagem de uma universidade estadual paulista. SMAD Revista Eletrônica Saúde Mental Álcool e Drogas, Ribeirão Preto, v. 2, n. 2, p. 12, 2006.

BANZATO, D. A.; KRONKA, S. N. Experimentação agricola. Jaboticabal: FUNEP, 1989. 247 p.

BARBOZA, R. A. B.; MENEGHIN, M. C.; SANTOS, V. R.; FONSECA, S. A.; FARIA, J. B. Efeito do envelhecimento na qualidade da cachaça produzida por pequenos produtores. Revista Ciência em Extensão, São Paulo, v. 6, n. 2, p. 46, 2010.

BARROS, M. B. A.; MARÍN-LEÓN, L.; OLIVEIRA, H. B.; DALGALARRONDO, P.; BOTEGA, N. J. Perfil do consumo de bebidas alcoólicas: diferenças sociais e demográficas no Município de Campinas, Estado de São Paulo, Brasil, 2003. Epidemiol. Serv. Saúde, Brasília, v. 17, n. 4, p. 259-270, 2008.

CARNEIRO, J. D. S. Estudo dos fatores da embalagem $e$ do rótulo de cachaça no comportamento dos consumidores. 2007. Tese (Doutorado em Ciência e Tecnologia de Alimentos), Universidade Federal de Viçosa, Viçosa.

COSTA, F. J.; CAVAlCANTE, A. A. Comportamento do consumidor de cerveja: proposta de uma tipologia baseada na imagem e nas intenções em relação à marca. Revista de Negócios, Blumenau, v. 12, n. 4, p. 71-85, out./dez. 2007.

COUTINHO, E. P. Dinâmica da modernização do setor de produção de aguardente de cana-de-açúcar no Brasil: construindo uma cachaça de qualidade. 2001. Tese (Doutorado em Engenharia da Produção) - Universidade Federal do Rio de Janeiro, Rio de Janeiro.
EVARISTO, R.A.; NÓBREGA, I. C. C. Perfil tecnológico de engenhos de cachaça do Estado da Paraíba. In: JORNADA NACIONAL DA AGROINDÚSTRIA, 1., 2006, Bananeiras. Anais... Bananeiras: UFPB, 2006. 6p.

FERNANDES, A. P.; SANTOS, M. C.; LEMOS, S. G.; FERREIRA, M. M. C.; NOGUEIRA, A. R. A; NÓBREGA, J. A. Pattern recognition applied to mineral characterization of Brazilian coffees and sugar-cane spirits. Spectrochimica Acta Part B, Mar del Plata, v. 60, n. 1, p. 717-724, 2005.

FIGUEIREDO FILHO, D.; SILVA JUNIOR, J. Desvendando os mistérios do coeficiente de correlação de Pearson (r). Revista Política Hoje, América do Norte, v. 18, n. 1, jan. 2009. Disponível em: <http://www.ufpe. br/politicahoje/index.php/politica/article/view/6/6>. Acesso em: 18 fev. 2011.

INSTITUTO BRASILEIRO DA CACHAÇA - IBRAC. Mercado interno. 2010. Disponível em <www. ibraccachacas.org.>. Acesso em 19 jul. 2011.

KOTLER, P.; KELLER, K. L. Administração de marketing. 12. ed. São Paulo: Pearson Prentice Hall, 2006. $750 \mathrm{p}$.

LARANJEIRA, R.; PINSKY, I.; ZALESKI, M.; CAETANO, R. I. Levantamento Nacional sobre os padrões de consumo de álcool na população brasileira. Revisão técnica científica: Paulina do Carmo Arruda Vieira Duarte. Brasília: Secretaria Nacional Antidrogas, 2007.

LOUREIRO, I. M. A. L. $O$ ambiente informacional na aplicação do regulamento de avaliação da conformidade do Inmetro nas empresas: um estudo do segmento da cachaça. 2008. Dissertação (Mestrado em Ciência da Informação) - Instituto Brasileiro de Informação em Ciência e Tecnologia. Universidade Federal Fluminense, Niterói.

MASCARENHAS, M. D. M.; MALTA, D. C.; SILVA, M. M. A.; CARVALHO, C. G.; MONTEIRO, R. A.; NETO, O. L. M. Consumo de álcool entre vítimas de acidentes e violências atendidas em serviços de emergência no Brasil, 2006 e 2007. Ciência \& Saúde Coletiva, Rio de Janeiro, v. 14, n. 5, p. 1789-1796, 2009.

MATSUNAGA, P.H.Identificação de atributos sensoriais de pedaços empanados de frango mais valorizados pelo consumidor. 2007. Dissertação (Mestrado em Alimentos e Nutrição) - Faculdade de Engenharia de Alimentos. Universidade Estadual de Campinas, Campinas.

NORONHA, R. L. F.; DELIZA, R.; SILVA, M. A. A. P. A Expectativa do consumidor e seus efeitos na avaliação sensorial e aceitação de produtos alimentícios. Alimentos e Nutrição, Araraquara, v. 16, n. 3, p. 299-308, jul./set. 2005. 
PEREIRA, A. F. Suplementação de nitrogênio sobre a fermentação alcoólica para produção de cachaça, cerveja e vinho. 2007. Dissertação (Mestrado em Ciência e Tecnologia de Alimentos) - Universidade Federal da Viçosa, Viçosa.

PRIBANIC, M. A. A cachaça capixaba: um estudo sobre barreiras à internacionalização. 2011. Dissertação (Mestrado em Administração) - Fundação Instituto Capixaba de Pesquisa em Contabilidade, Economia e Finanças, Vitória.

REIS, G. V. O consumo de bebida alcoólica entre alunos do ensino fundamental e médio. 2008. Trabalho de Conclusão de Curso (Curso de Graduação em Enfermagem) - Universidade Paranaense, Paranavaí.

SAMPAIO, I. B. M.Estatística aplicada à experimentação animal. Belo Horizonte: FEPMVZ, 1998. 221 p.

SAS Institute Inc. SAS/STAT User's guide. Version 6.12. Cary, North Caroline: SAS Institute Inc., 1996.
SILVA, M. J. Percepção da qualidade da cachaça pelo consumidor: notoriedade das marcas versus aceitação sensorial. 2011. Dissertação (Mestrado em Tecnologia Agroalimentar) - Universidade Federal da Paraíba, Bananeiras.

STRAUCH, E. S.; PINHEIRO, R. T.; SILVA, R. A.; HORTA, B. L. Uso de álcool por adolescentes: estudo de base populacional. Revista Saúde Pública, São Paulo, v. 43, n. 4, p. 647-55, 2009.

VIDAL, M. F.; GONÇALVES, M. F. Produção de cachaça na área de jurisdição do BNB: mercado e estrutura da cadeia produtiva. In: CONGRESSO BRASILEIRO DA SOCIEDADE DE ECONOMIA, ADMINISTRAÇÃO E SOCIOLOGIA RURAL, 46., 2008, Rio Branco, Acre. Anais... Rio Branco, Acre: FAAO, 2008. p. 21.

VIEIRA, J. R. P.; PEREIRA, E. L. Avaliação do perfil de consumo de vinho de frequentadores de bares noturnos no Município de Cascavel/PR. 2006. Disponível em: $<\mathrm{http}: / /$ www.fag.edu.br/graduacao/nutricao/resumos2006>. Acesso em: 05 out. 2010. 
